Structure transitions induced by the Hal I term i $n$ honogeneous and i sot ropi $c$ magnet ohydr odynami c t ur bul ence

\begin{tabular}{|l|l|}
\hline $\begin{array}{l}\text { j our nal or } \\
\text { publ i cat i on titl e }\end{array}$ & Physi cs of Pl asmas \\
\hline vol une & Vol . 21 \\
\hline page range & $072313-1$ - 10 \\
\hline year & 2014 $01-01$ \\
\hline URL & ht t p: //hdl . handl e. net /10655/12477 \\
\hline
\end{tabular}




\section{AIP $\left.\right|_{\text {Physics of }}$

\section{Structure transitions induced by the Hall term in homogeneous and isotropic magnetohydrodynamic turbulence}

H. Miura and K. Araki

Citation: Physics of Plasmas (1994-present) 21, 072313 (2014); doi: 10.1063/1.4890857

View online: $\mathrm{http}: / / d x . d o i . o r g / 10.1063 / 1.4890857$

View Table of Contents: http://scitation.aip.org/content/aip/journal/pop/21/7?ver=pdfcov

Published by the AIP Publishing

\section{Articles you may be interested in}

Structures and dynamics of small scales in decaying magnetohydrodynamic turbulence

Phys. Fluids 25, 105106 (2013); 10.1063/1.4824195

Intermittency and geometrical statistics of three-dimensional homogeneous magnetohydrodynamic turbulence: A wavelet viewpoint

Phys. Plasmas 18, 092304 (2011); 10.1063/1.3628637

Electromagnetically driven dwarf tornados in turbulent convection

Phys. Fluids 23, 015103 (2011); 10.1063/1.3541817

Wavelet-based coherent vorticity sheet and current sheet extraction from three-dimensional homogeneous magnetohydrodynamic turbulence

Phys. Plasmas 16, 082306 (2009); 10.1063/1.3195066

A numerical study of the alpha model for two-dimensional magnetohydrodynamic turbulent flows

Phys. Fluids 17, 035112 (2005); 10.1063/1.1863260

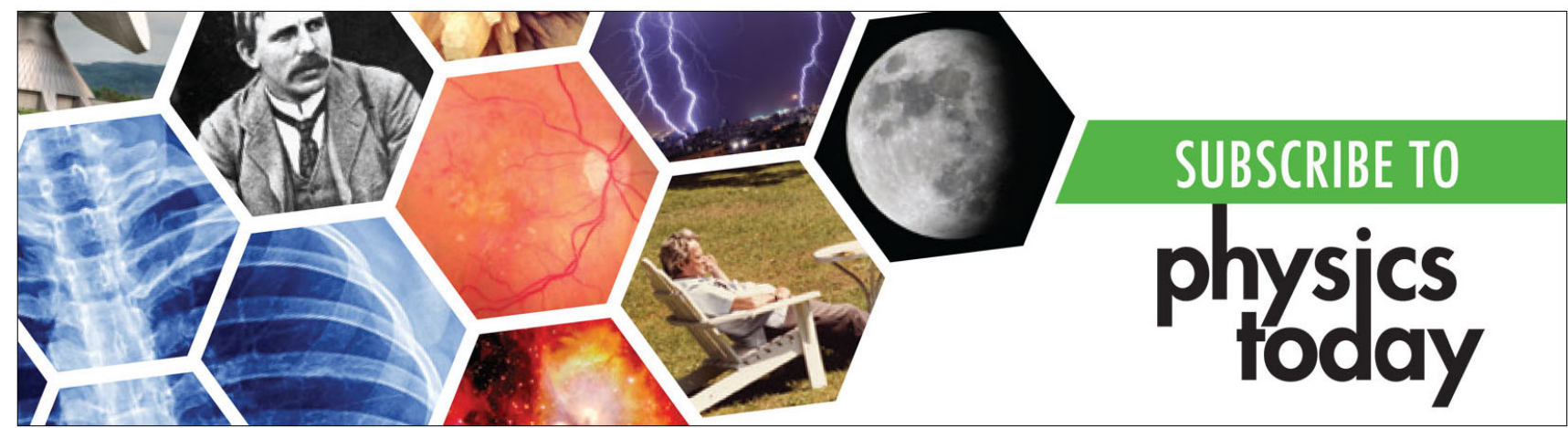




\title{
Structure transitions induced by the Hall term in homogeneous and isotropic magnetohydrodynamic turbulence
}

\author{
H. Miura ${ }^{1, a)}$ and K. Araki ${ }^{2}$ \\ ${ }^{1}$ Department of Helical Plasma Research, National Institute for Fusion Science, 322-6 Oroshi, \\ Toki, Gifu 509-5292, Japan \\ ${ }^{2}$ Faculty of Engineering, Okayama University of Science, 1-1 Ridai-cho, Okayama 700-0005, Japan
}

(Received 26 May 2014; accepted 10 July 2014; published online 23 July 2014)

\begin{abstract}
Hall effects on local structures in homogeneous, isotropic, and incompressible magnetohydrodynamic turbulence are studied numerically. The transition of vortices from sheet-like to tubular structures induced by the Hall term is found, while the kinetic energy spectrum does not distinguish the two types of structures. It is shown by the use of the sharp low-pass filter that the transition occurs not only in the scales smaller than the ion skin depth but also in a larger scale. The transition is related with the forward energy transfer in the spectral space. Analyses by the use of the sharp low-pass filter show that the nonlinear energy transfer associated with the Hall term is dominated by the forward transfer and relatively local in the wave number space. A projection of the simulation data to a Smagorinsky-type sub-grid-scale model shows that the high wave number component of the Hall term may possibly be replaced by the model effectively. (C) 2014 AIP Publishing LLC.

[http://dx.doi.org/10.1063/1.4890857]
\end{abstract}

\section{INTRODUCTION}

Turbulence is one of the most important keywords in many categories of natural sciences and engineering such as astrophysics, fluid mechanics, and nuclear fusion. Homogeneous magnetohydrodynamic (MHD) turbulence provides a basic framework to study non-linearity, intermittency, and the statistical nature of turbulence. There have been numerous experimental, numerical, and theoretical works on turbulent energy spectrum, anisotropy, intermittency, and other topics of homogeneous MHD turbulence. ${ }^{1-8}$ The energy spectrum proportional to $k^{-5 / 3}$ ( $k$ is the wave number) is supported by solar wind observations. ${ }^{8-10}$ It has been reported on the satellite-observations that the magnetic energy spectrum has the $-5 / 3$ power-law and a succeeding region of a sharper slope refracting at the scale of the ion skin depth. There are also reports on the statistical natures of turbulence such as the structure functions. Matthaeus et al. ${ }^{26}$ have reported regarding the alignment of velocity and magnetic field in (single-fluid) MHD turbulence. Servidio et al. ${ }^{27}$ have reported on the depression of non-linearity in isotropic MHD turbulence due to the alignment of vectors. These studies have provided some clear images of the statistical nature of MHD turbulence.

While MHD turbulence has been studied extensively, extensions of MHD equations to two-fluid effects attract much attention. ${ }^{11,12}$ Though MHD equations provide a good platform to study plasma turbulence in macroscopic scales, the lack of certain effects which are related to the ion skin depth and the Larmor radius can make studies in small scales inaccurate. One of the simplest approaches to improve the accuracy of the model is introducing the Hall term to the MHD equations. The Hall term is expected to change the energy spectrum by introducing the scale of the ion skin

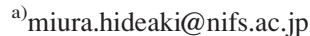

depth explicitly into the set of equations. The Hall term is also believed to enhance the magnetic reconnection and change various turbulent natures. The influences of the Hall term on turbulent energy spectrum, energy transfer, dynamo action, structure functions, and other topics have been studied. $^{22-24,27}$ The Hall term is also considered to change the formation of turbulent structures through changing natures of instability which often occur prior to the structure formation. It has been shown that the Hall effect can either enhance or suppress various instability, such as the Rayleigh-Taylor and Kelvin-Helmholtz instability, depending on the parameters. ${ }^{13-15}$

Turbulent structures, or local structures here, is important especially because it is an origin of the intermittency and the non-Gaussian nature of turbulent statistics. Local structures are formed as a consequence of nonlinear dynamics. Hence, observations of the structures enable us to elucidate what kind of dynamics is dominant there. As for the local structures, tubular vortices are one of the representative local structures in homogeneous neutral-fluid turbulence. $^{16-18}$ In single-fluid MHD turbulence, the current sheets are often reported in MHD turbulence. ${ }^{19-21}$ Furthermore, electric field visualization in Hall MHD turbulence has been reported by Dmitruk and Matthaeus. ${ }^{25}$ Intermittency in Hall MHD turbulence has been recently studied by Rodriguez Imazio et al. ${ }^{28}$ Reduction of nonlinearity has been also studied by the authors ${ }^{29}$ from the points of view of the energy transfer functions. In spite of these works, elementary structures and their impact on the statistical nature of Hall MHD turbulence are not clarified yet.

In this paper, we focus on the Hall effects on local structures in freely decaying, homogeneous, isotropic, and incompressible Hall MHD turbulence. Though the assumption of the isotropy is too strong to apply to the studies of solar winds and nuclear fusion, this assumption enables us to 
make use of accumulated knowledge on turbulence structures. This paper is organized as follows. In Sec. II, the outlines of Hall MHD turbulence are shown. In Sec. III, the transition of structures induced by the Hall term is reported. Some statistical nature in Hall MHD turbulence is also reported. In Sec. IV, we study scale-range on which the Hall term influences are studied. In Sec. V, the nature of high wave number components in the turbulent field is examined by projecting them to a classical Smagorinsky-type sub-gridscale model. Summary is presented in Sec. VI.

\section{DIRECT NUMERICAL SIMULATIONS (DNS)}

DNS of homogeneous and isotropic decaying Hall MHD turbulence is performed by solving the equations

$$
\begin{gathered}
\frac{\partial u_{i}}{\partial t}=-\frac{\partial\left(u_{i} u_{j}\right)}{\partial x_{j}}-\frac{\partial p}{\partial x_{i}}+\epsilon_{i j k} J_{j} B_{k}+\frac{1}{R e_{\nu}} \frac{\partial^{2} u_{i}}{\partial x_{j} \partial x_{j}} \\
\frac{\partial u_{i}}{\partial x_{i}}=0 \\
\frac{\partial B_{i}}{\partial t}=\epsilon_{i j k} \frac{\partial}{\partial x_{j}}\left[\epsilon_{k m n}\left(u_{m}-\epsilon_{H} J_{m}\right) B_{n}\right]+\frac{1}{R e_{\eta}} \frac{\partial^{2} B_{i}}{\partial x_{j} \partial x_{j}} \\
\frac{\partial B_{i}}{\partial x_{i}}=0
\end{gathered}
$$

numerically, where $u_{i}, B_{i}$, and $J_{i}=\epsilon_{i j k} \partial_{j} B_{k}$ are the $i$-th components of the velocity, the magnetic field, and the current density vectors, respectively. The summation convention is applied for repeated suffixes. The symbols $\epsilon_{i j k}, \epsilon_{H}, R e_{\nu}, R e_{\eta}$ denote the Levi-Civita symbol, the Hall parameter (the ion skin depth normalized by the system size), the Reynolds number, and the Lundquist number, respectively. These equations are already normalized by some reference quantities. The Hall MHD equations are solved numerically by the pseudo-spectral method and the Runge-Kutta-Gill scheme under the triply periodic boundary condition over a $(2 \pi)^{3}$ cube. The number of grid points is $N^{3}=1024^{3}$. The aliasing errors are removed by the 2/3-rule. Thus, the maximum wave number $k_{\max }=341$. The initial condition is provided so that the spectra of the velocity and the magnetic field are proportional to $k^{2} \exp \left(-k^{2} / k_{0}^{2}\right) \quad\left(k_{0}=2\right.$ here $)$ with random phases. Initially the kinetic and magnetic energies are almost the same. The Hall parameter is varied $\epsilon_{H}=0,0.0125,0.025$, and 0.05, while the reference Reynolds and Lundquist numbers are kept $R e_{\nu}=R e_{\eta}=2000$ throughout this paper. Since all the parameters except the Hall parameter are the same among all the simulations, every single difference among them are attributed to the Hall effect. See also our earlier results with smaller numbers of grid points ${ }^{30-32}$ for some basic nature of turbulence evolution.

The development of turbulence in a freely decaying simulation can be seen quickly by observing the growth of the enstrophy $Q_{\omega}=\left\langle\omega_{m} \omega_{m}\right\rangle / 2$ and the total current $Q_{J}=\left\langle J_{m} J_{m}\right\rangle / 2$, where $\omega_{i}=\epsilon_{i j k} \partial u_{k} / \partial x_{j}$ is the $i$-th component of the vorticity vector. The enstrophy $Q_{\omega}$ is one of the simplest measures for seeing the development of turbulence in decaying neutral-fluid turbulence. Here, we also observe $Q_{J}$ as the counterpart of $Q_{\omega}$ in the magnetic field. In Fig. 1, the time evolution of (a) $Q_{\omega}$ and (b) $Q_{J}$ are plotted for the four $\epsilon_{H}$ s. Both $Q_{\omega}$ and $Q_{J}$ start from relatively low values and evolve to high values. They peak at $t \simeq t_{P}=0.6$ (in the unit of the eddy-turn-over time) and decay. It is noted that the Taylor-scale Reynolds number starts from about 1600 and decay monotonically to about 90 both in the velocity and in the magnetic field. The plots show that $Q_{\omega}$ is suppressed when $\epsilon_{H}$ is increased while $Q_{J}$ is increased for increasing $\epsilon_{H}$ in the evolving stage of decaying turbulence $t \leq 0.5$. A persistent nature in Fig. 1 over $\epsilon_{H} \mathrm{~s}$ is that $Q_{J}>Q_{\omega}$. See our previous work ${ }^{30}$ for the kinetic and magnetic energy decay.

Another measure of the development of turbulence is the energy spectra normalized by the Kolmogorov length scale. In Fig. 2, (a) the kinetic energy spectrum $E_{K}\left(k a_{\nu}\right) / \nu U_{\nu}^{2}$ and (b) the magnetic energy spectrum $E_{M}\left(k b_{\eta}\right) / \eta B_{\eta}^{2}$ at $t=2.4$ are shown. Here, the symbols $\epsilon_{\nu}, a_{\nu}=\left(\nu^{3} / \epsilon_{\nu}\right)^{1 / 4}$, and $U_{\nu}=\left(\nu \epsilon_{\nu}\right)^{1 / 4}$ are the kinetic energy dissipation rate, the Kolmogorov length scale, and the velocity at the Kolmogorov length scale, respectively, while the symbol $\epsilon_{\eta}, b_{\eta}=\left(\eta^{3} / \epsilon_{\eta}\right)^{1 / 4}$ and $B_{\eta}=\left(\eta \epsilon_{\eta}\right)^{1 / 4}$ are their counterparts in the magnetic field. The spectra of $\epsilon_{H}=0.0125$ are
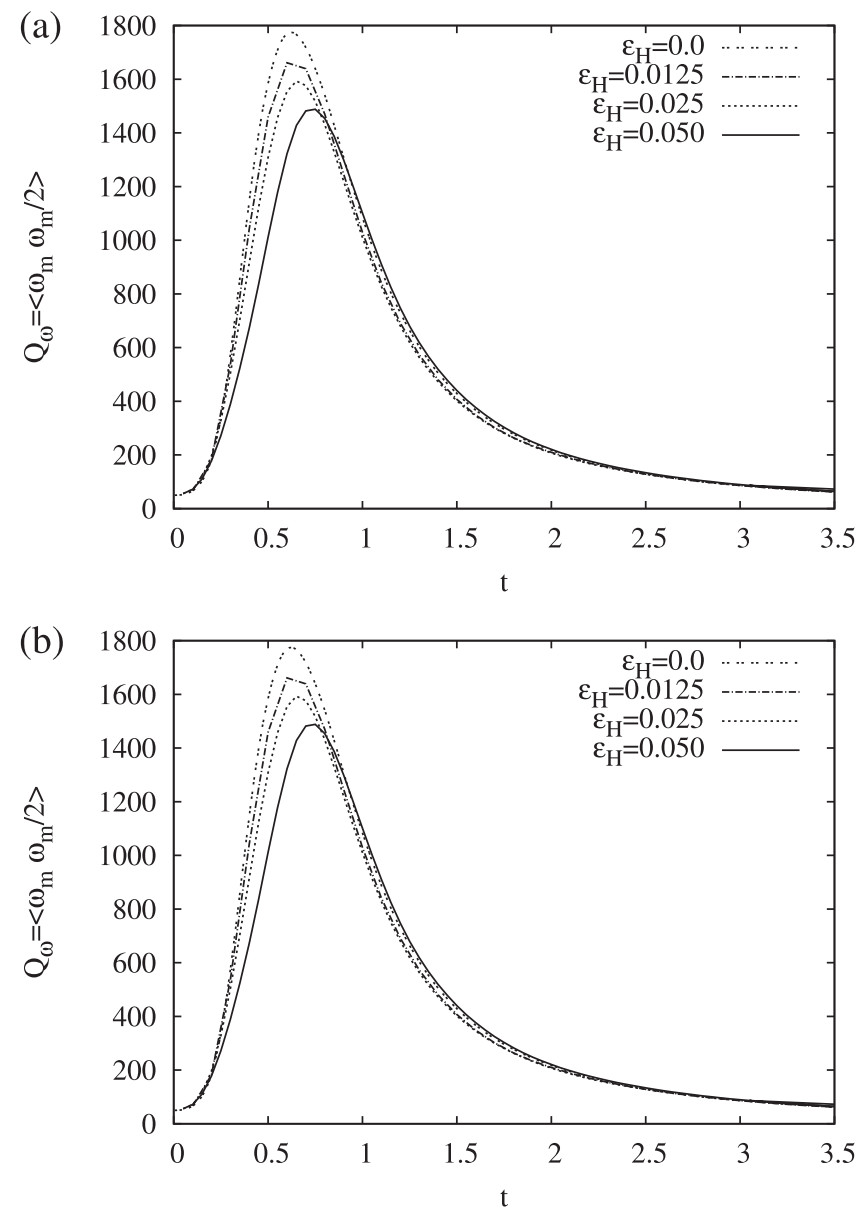

FIG. 1. Time evolution of (a) the enstrophy density and (b) the total current. Dependence of the quantities on $\epsilon_{H}$ is just the opposite between (a) and (b). The abscissa is the time normalized by the initial eddy turn over time $\left(u_{0} k_{0}\right)^{-1}$. 
(a)

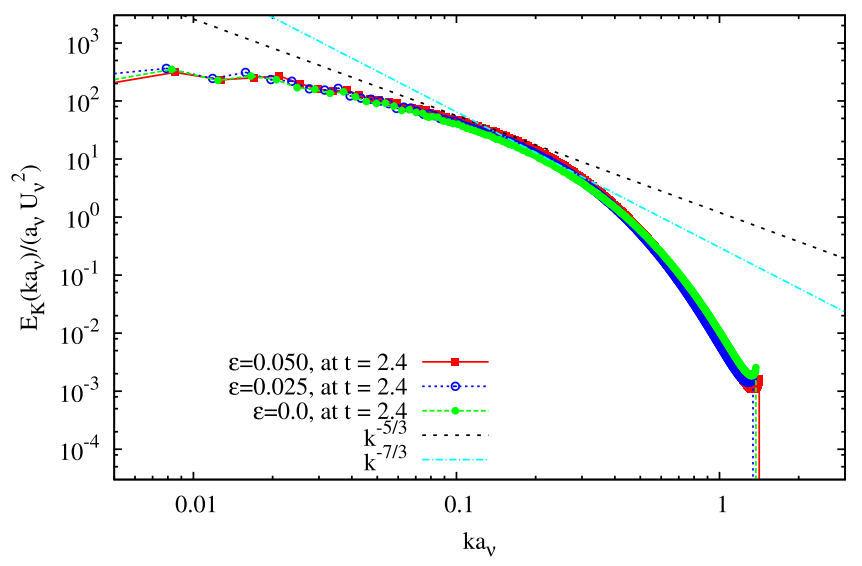

(b)

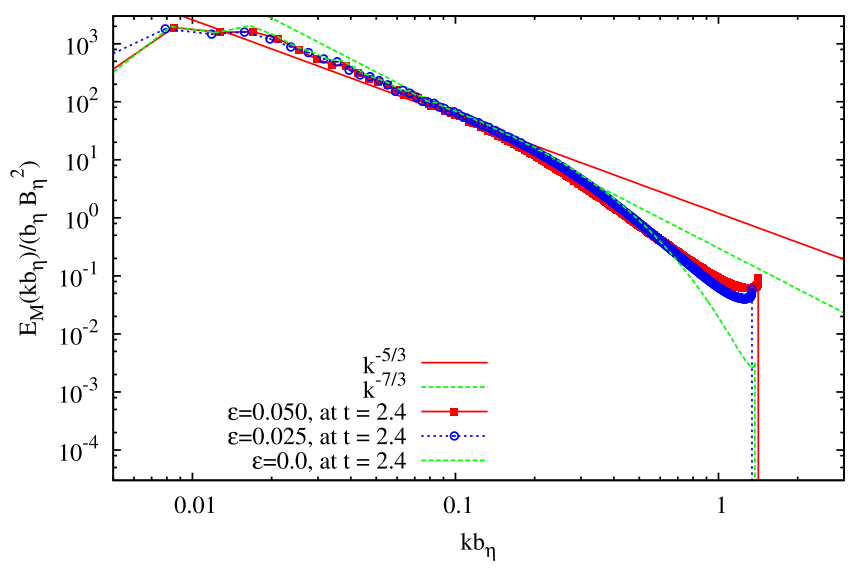

FIG. 2. The dependence of (a) the kinetic energy spectrum and (b) the magnetic energy spectrum on the $\epsilon_{H}$. The spectra are normalized by the use of the Kolmogorov length scale.

omitted for the clarity of the plots. It can be shown that the normalized spectra at each time after $t \simeq 2$ (a few times of $\left.t_{P}\right)$ are collapsed to those shown in Fig. 2. The solid and dashed lines represent $k^{-5 / 3}$ and $k^{-7 / 3}$, respectively. The scale of the ion skin depth $k_{H}=1 / \epsilon_{H}$ is at $k a_{\nu} \simeq k b_{\eta} \simeq 0.1$. It has been reported earlier by numerical simulations ${ }^{30,35}$ that the energy spectra for $k>k_{H}$ is likely to be scaled by $k^{-7 / 3}$. Spectra in Fig. 2(b) may be near to $k^{-7 / 3}$, being consistent both with other numerical simulations $\mathrm{s}^{24,34,35}$ and with the observations in solar winds, ${ }^{9,10}$ although the power-law index is not our concern here.

Notice here that the magnetic energy is larger than the kinetic energy at the time in Fig. 2, because the kinetic energy is transferred away from its lower wave number components of about $k a_{\eta}<0.4$ in the course of the decaying simulations while the magnetic energy at the wave number region is supplied either by the nonlinear transfer or by the energy exchange between the kinetic and magnetic energy. The dominance of the magnetic energy over the kinetic energy is the consequence of the energy exchange between the two energies. ${ }^{30}$ It is noted here that, since the spectral tail in Fig. 2(b) becomes less steep for a larger $\epsilon_{H}$, the introduction of the Hall term causes a stronger magnetic diffusion for larger Hall parameter, and leads to faster decay of $J$ as in Fig. 1(b). From the observations in Figs. 1 and 2, influences
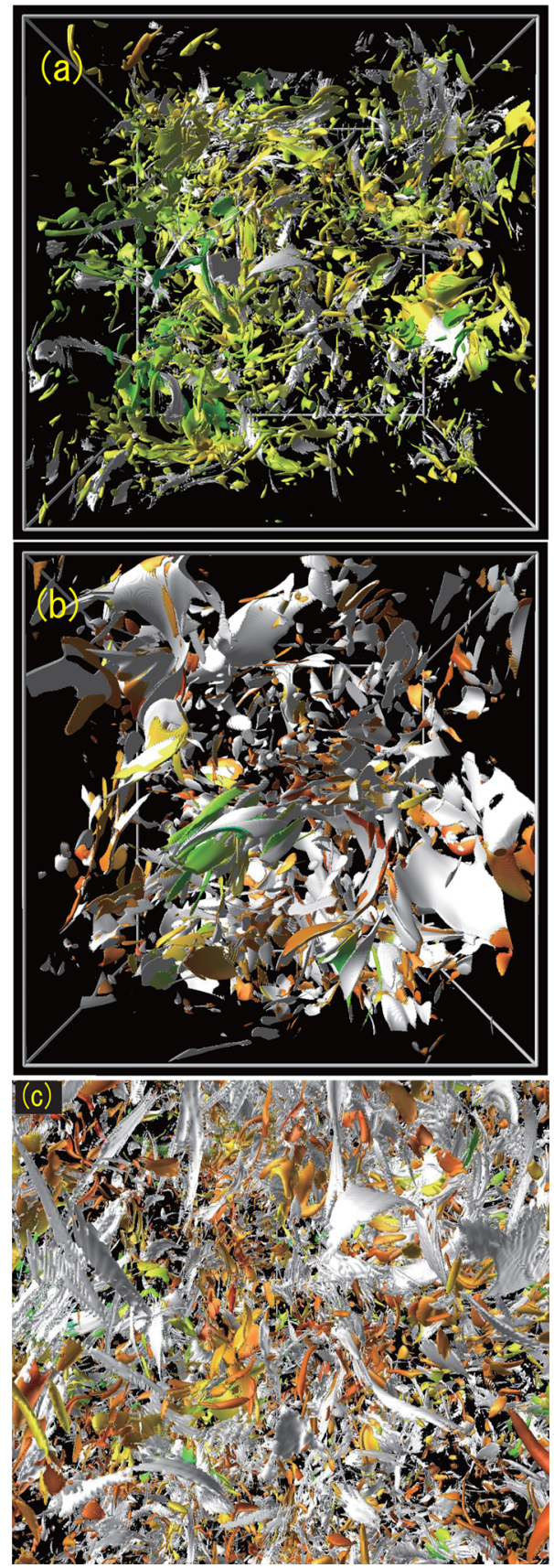

FIG. 3. Isosurfaces of the enstrophy density (colored) and the current density (grey) in (a) Hall MHD turbulence with $\epsilon_{H}=0.05$ and (b) single-fluid MHD turbulence $\left(\epsilon_{H}=0\right)$ for $512^{3}$ grid points out of the entire $1024^{3}$ grid points. (c) Close-up of the isosurfaces of the enstrophy density and the current density drawn by the use of full $1024^{3}$ grid points. The thresholds of the isosurfaces are given as about five times of the deviation larger above the average value for $q_{\omega}$ and $q_{J}$, respectively. The colors of the $q_{\omega}$ isosurfaces represent the ratio of the kinetic energy to the magnetic energy.

of the Hall term appear mostly in the magnetic field rather than in the velocity field. However, in Sec. III, it turns out that a larger qualitative change is observed more in local structures of the velocity field (or the vorticity field, equivalently).

\section{LOCAL STRUCTURES}

Local structures, such as sheets and tubes of vortices, are formed as the consequence of dynamical evolution 
and are considered the source of the intermittency of turbulence. Here, two typical quantities, enstrophy density $q_{\omega}$ $=\omega_{m} \omega_{m} / 2$ and the current density $q_{J}=J_{m} J_{m} / 2$, are observed. In Fig. 3(a), isosurfaces of $q_{\omega}$ and $q_{J}$ in Hall MHD turbulence of $\epsilon_{H}=0.05$ are shown at $t=2.4$. Isosurfaces in Fig. 3(b) are for single-fluid MHD turbulence $\left(\epsilon_{H}=0\right)$ at the same time. The colored isosurfaces are for $q_{\omega}$ and the grey isosurfaces are for $q_{J}$. In both Figs. 3(a) and 3(b), a region of $512^{3}$ grid-points is presented out of the total grid number $1024^{3}$. It is easily observed that the isosurfaces in Fig. 3(a) are a collection of very fine structures, scattered over the presented region almost uniformly. In particular, many of isosurfaces of $q_{\omega}$ are tubular as we see later in Fig. 3(c) more clearly. In contrast to Fig. 3(a), the isosurfaces in Fig. 3(b) consist of flattened sheets which cover wide areas in the box, the generation of which is well-known. ${ }^{19,21}$ The sheets of $q_{\omega}$ (vortex sheets) and $q_{J}$ (current sheets) almost stay together with each other, making a contrast to the visualization in Fig. 3(a). It should be recalled that all differences among DNS in this paper are attributed to the difference of $\epsilon_{H}$. Thus, the change of the structure is attributed to the Hall parameter, too.

In Fig. 3(c), the isosurfaces of $q_{\omega}$ and $q_{J}$ are shown with full $1024^{3}$ grid points more closely than Fig. 3(a). It is clearly observed that many of the colored isosurfaces for $q_{\omega}$ are tubular, and some of the $q_{\omega}$ isosurfaces are curved sheets which can look as if they are going to roll up to tubes. It is also found that the grey isosurfaces for $q_{J}$ remain in sheetstructures although they are torn into small sheets. The generation of smaller sheets is quite natural because of the enhancement of high wave number coefficients of the magnetic field due to the Hall effect. It is also thought that the separation of isosurfaces of $q_{\omega}$ from those of $q_{J}$ is helped by the change of the frozen-in condition due to the Hall term as well as by the magnetic reconnection which should happen more frequently than the single-fluid MHD case. Recall that in contrast to the magnetic energy spectra the kinetic energy spectra in Fig. 2(a) do not show a clear difference for different $\epsilon_{H}$ s. In other words, the isotropic energy spectrum does not distinguish tubular vortex structures from sheet-like structures. We remark here that the visualization of the electric field does not show a sharp difference between the Hall and single-fluid MHD turbulence as we see in Figs. 3(a) and

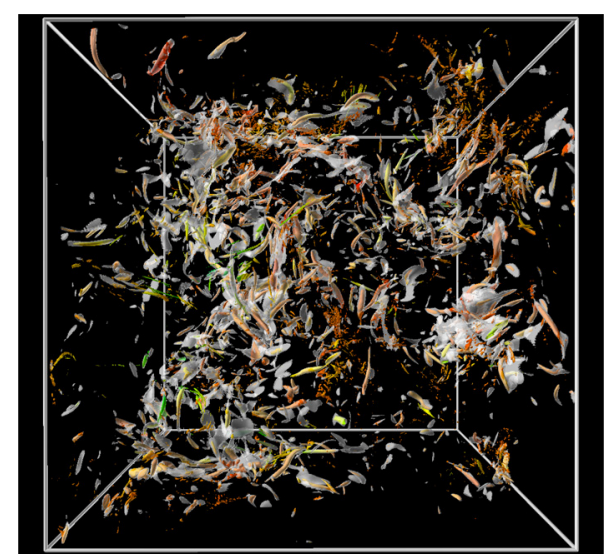

FIG. 4. Isosurfaces of the enstrophy density (colored) and the Laplacian of the magnetic pressure (semi-transparent grey) in Hall MHD turbulence with $\epsilon_{H}=0.05$. 3(b), although the electric field is one of the key variables to distinguish the two kinds of turbulent field. A study of the impact of the structure transition on the electric field is left as one of future works.

The tubular vortices in Hall MHD turbulence also can be characterized by the Laplacian of the magnetic pressure $P_{M}=B_{i} B_{i} / 2$. In Fig. 4, isosurfaces of $P_{M}$ are drawn in semitransparent grey together with the colored isosurfaces of the enstrophy density. Isosurfaces of both $P_{M}$ and the enstrophy density are tubular, overlapping with each other at everywhere in the space. A comparison of the structures with those in hydrodynamic turbulence can give an understanding about this coincidence. A tubular vortex in hydrodynamic turbulence usually consists of a low-pressure region on the cross-section (swirling-plane) of the vortex perpendicular to the vortex axis ${ }^{17,37-39}$ in order to take a counter-balance to the centrifugal force caused by the swirling motion of the vortex. The low-pressure nature on the swirling plane is characterized by the two negative eigenvalues of the pressure Hessian, $\partial^{2} p / \partial x_{i} \partial x_{j}$. On this plane, the Laplacian of the pressure which is equal to the sum of the three eigenvalues of the Hessian tends to have a large negative value. This is the reason why the negative threshold of the Laplacian gives the visualization of tubular vortices. In contrast to hydrodynamic turbulence, the magnetic pressure analyzed here is a few times larger than the hydrodynamic pressure, due to the dominance of the magnetic energy over the kinetic energy. Thus, the magnetic field is more responsible to the vortex structure formation than the velocity field itself. Fig. 4 indicates that the centrifugal force of swirling vortices is balanced by the low magnetic pressure, not by the low hydrodynamic pressure as in the vortices in neutral fluid turbulence. Recall that tubular vortices in neutral fluid turbulence develop typically from vortex sheets either by the Kelvin-Helmholtz (KH) instability or by the wrapping mechanism of perpendicular vorticity to the parallel vorticity. ${ }^{36}$ Thus, from the point of view of local dynamics, the appearance of tubular vortices suggests that the $\mathrm{KH}$ instability or the wrapping mechanism are allowed to work by breaking the frozen-in condition (in the sense of single-fluid MHD), which ties the vorticity and the current density fields closely.

\section{SCALE-DEPENDENCE OF VORTEX STRUCTURES}

A question can arise naturally from the analysis in Sec. III with regard to the range of the interaction: how is the scale of the Hall term, which is the ion skin depth, related with the structure transition? Sought here is the scale-range of the transition in order to answer the questions (1) whether the Hall term influences the scales smaller than the ion-skin-depth scale or the scales larger than it, and (2) whether the interaction is local or non-local in the wave number space. From our earlier studies on the energy transfer functions, the nonlinear interaction associated with the Hall term appears local in the wave number space although there can be some strong interaction between the moderate and low wave number regions. ${ }^{33}$

In Fig. 5(a), isosurfaces of $q_{\omega}$ and $q_{J}$ in $\epsilon_{H}=0.05$ Hall MHD turbulence are drawn, retaining Fourier components up to the maximum wave number $N / 3=341$. In Figs. 
(a)

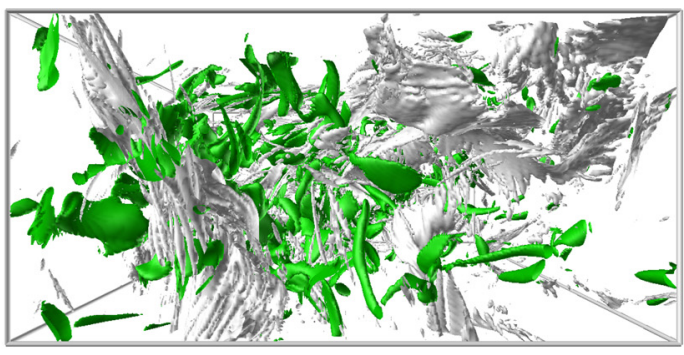

(b)

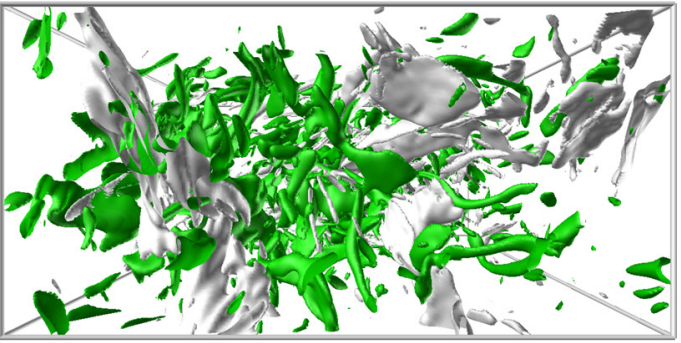

(c)

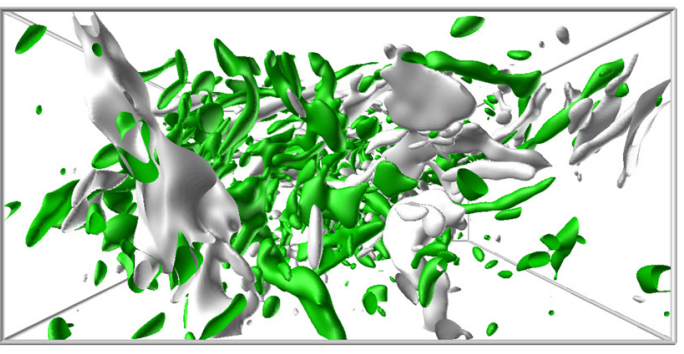

(d)

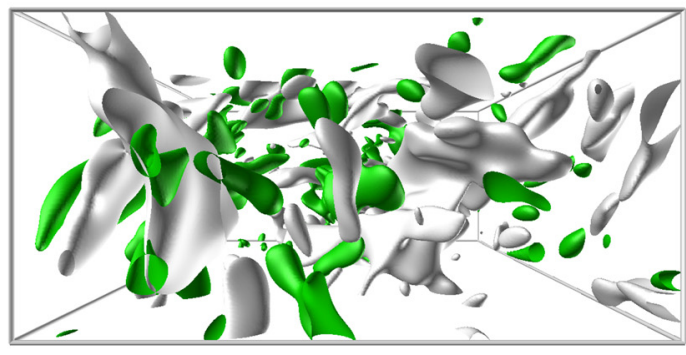

FIG. 5. Isosurfaces of the current density and the enstrophy density in Hall MHD turbulence $\left(\epsilon_{H}=0.05\right)$ (a) without the low-pass filter, and those with the low-pass filter of the cut-off wave number (b) $k_{c}=128$, (c) $k_{c}=64$, and (d) $k_{c}=32$.

5(a)-5(c), isosurfaces of $q_{\omega}$ and $q_{J}$ are drawn with the sharp low-pass filter of the cut-off wave number (b) $k_{c}=128$, (c) $k_{c}=64$, and (d) $k_{c}=32$, respectively, operated to $\omega_{i}$ and $J_{i}$. Here, the sharp low-pass filter means truncating the Fourier coefficients of $k>k_{c}$. Figure 6 is their counterpart in singlefluid MHD turbulence. In Fig. 5, tubular vortices are observed in every panel, while the population and location of the vortices are somewhat different among the panels. The difference of the population and location of tubular vortices among Figs. 5(a)-5(d) shows a scale-hierarchy of tubular vortices: there are tubular vortices at each scale and they appear sometimes at different locations depending on the cut-off wave number of the low-pass filter. It is also observed that the current density looks sheet-like at each scale. This means that sheet-like structures similar to those in the singlefluid MHD turbulence are maintained in relatively low wave number components even though the isosurfaces of $q_{J}$ in Fig. 5 (a) is torn into pieces because of the excitation of the high wave number components. (a)

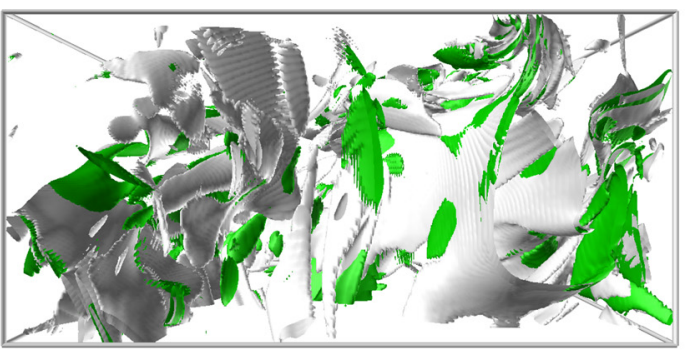

(b)

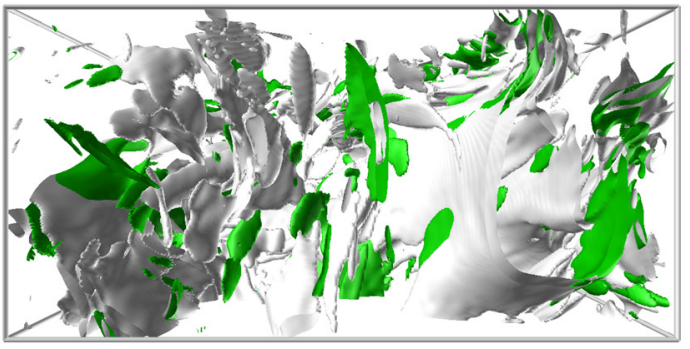

(c)

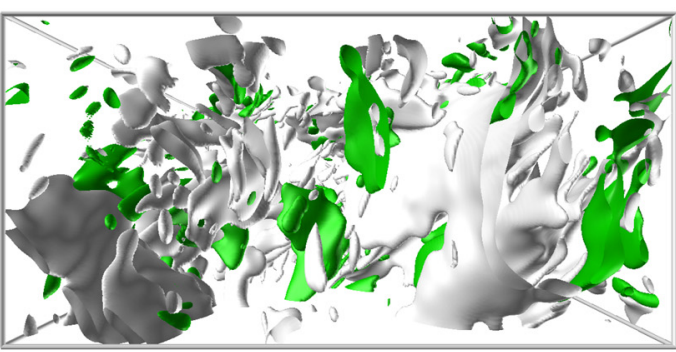

(d)

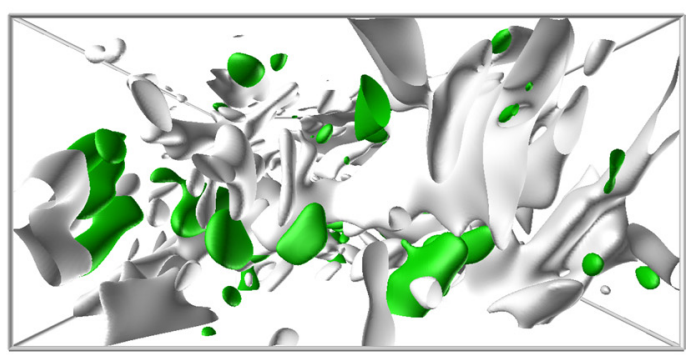

FIG. 6. Isosurfaces of the current density and the enstrophy density in the single-fluid MHD, corresponding to those in Fig. 5.

Fig. 5 shows that vortex tubes appear in all the cases (a)-(d). Since $k_{c}=32>k_{H}$, the observation in Fig. 5 for the influences of the Hall term to the scales smaller than the ion skin depth. Then, it can be interesting to see how the scales larger than the ion skin depth are affected by the Hall term. Hall MHD turbulence with $\epsilon_{H}=0.025$ gives us an access to study this point. In Fig. 7(a), the isosurfaces of $q_{\omega}$ and $q_{J}$ without the low-pass are drawn. There are fine structures both in the enstrophy density $q_{\omega}$ and the current density $q_{J}$. In Fig. 7(b), only isosurfaces of $q_{\omega}$ are magnified for a smaller region. It is observed that there are both the sheetlike and tubular isosurfaces. In Fig. 7(c), the isosurfaces of $q_{\omega}$ filtered by the low-pass filter of $k_{c}=32$ are shown. There are both the sheet-like and tubular isosurfaces even though $k_{c}=32<1 / \epsilon_{H}=20$. This means that the vortices can be tubular even when they consist of the Fourier components of the scales larger than the ion skin depth. Thus, the structure transition can occur not only for the scales smaller than the ion skin depth but also for the scales larger than the ion skin depth. The mixture of the vortex sheets and vortex tubes also suggests that the influences of the Hall term to the scales 
(a)

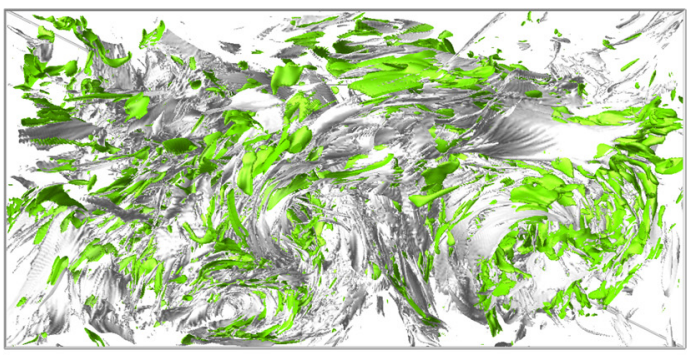

(b)

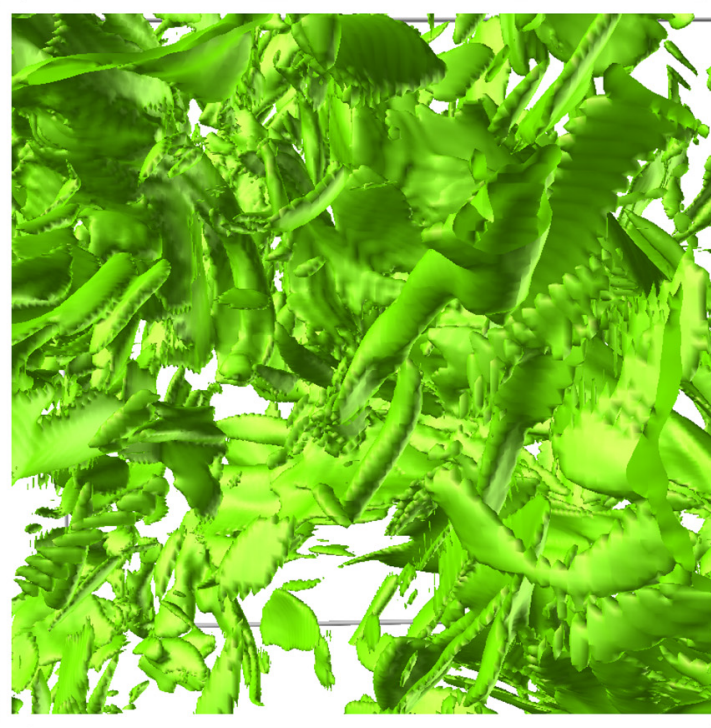

(c)

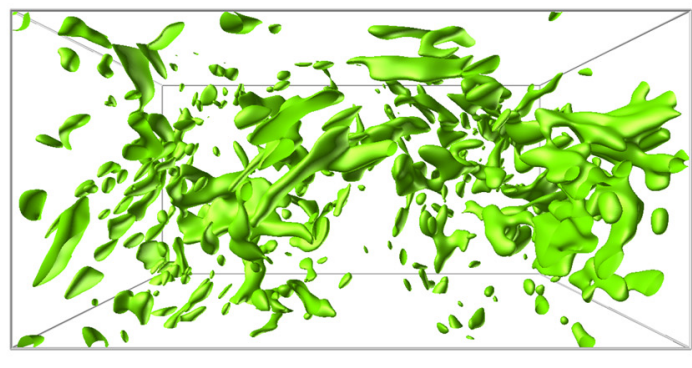

FIG. 7. Visualization in Hall MHD turbulence with $\epsilon_{H}=0.025$. (a) The isosurfaces of $q_{\omega}$ and $q_{J}$ without the low-pass filter. (b) A close-up of tubular vortices in (a). (c) The isosurfaces of the enstrophy density and the current density with the low-pass filter of $k_{c}=32$. A rectangular box of $512 \times 256 \times 256$ out of $1024^{3}$ grid points is used for the visualization.

larger than the ion skin depth can be relatively limited and local.

Aiming to study locality/non-locality of the Hall effects with regards to the structure transition, the energy transfer is examined by making use of the energy transfer functions $T_{K}(k)$ for the kinetic energy and $T_{M}(k)$ for the magnetic energy

$$
\begin{gathered}
T_{K}(k)=\sum_{[k]} \tilde{u}_{i}(\mathbf{k})^{*} \cdot F\left[-\frac{\partial\left(u_{i} u_{j}\right)}{\partial x_{j}}-\frac{\partial p}{\partial x_{i}}+\epsilon_{i j k} J_{j} B_{k}\right], \\
T_{M}(k)=T_{M}^{I}(k)+T_{M}^{H}(k), \\
T_{M}^{I}(k)=\sum_{[k]} \tilde{B}_{i}(\mathbf{k})^{*} \cdot F\left[\epsilon_{i j k} \frac{\partial}{\partial x_{j}}\left\{\epsilon_{k m n} u_{m} B_{n}\right\}\right], \\
T_{M}^{H}(k)=\sum_{[k]} \tilde{B}_{i}(\mathbf{k})^{*} \cdot F\left[\epsilon_{i j k} \frac{\partial}{\partial x_{j}}\left\{-\epsilon_{k m n} \epsilon_{H} J_{m} B_{n}\right\}\right] .
\end{gathered}
$$
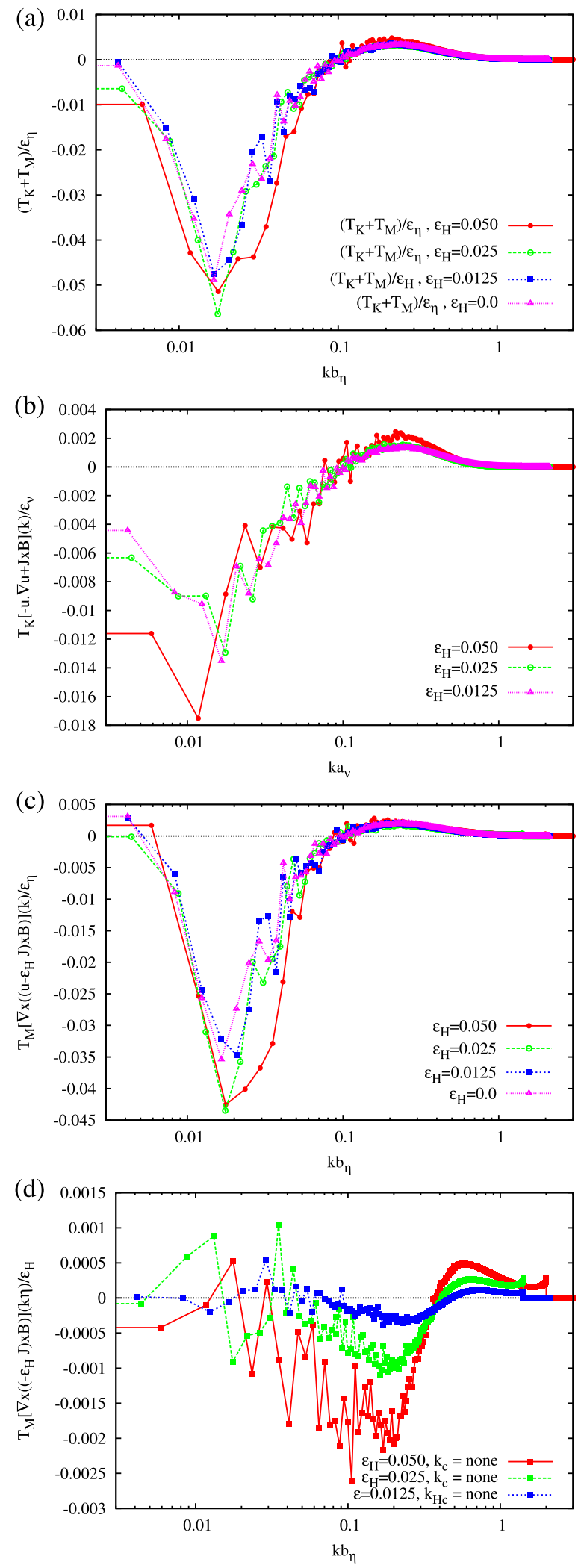

FIG. 8. Dependence of energy transfer functions on the Hall parameter $\epsilon_{H}$, (a) $T_{K}+T_{M}$, (b) $T_{K}$, (c) $T_{M}$, and (d) $T_{M}^{H}$.

Here, $\sum_{[\mathrm{k}]}$ and $\mathbf{k}$ represent the shell-average in the isotropic wave number space and the three-dimensional wave number vector, respectively. The symbols $F, \tilde{,}$, and * denote the Fourier transform, a Fourier coefficient, and the complex conjugate, respectively. In Fig. 8, energy transfer functions 
(a) $T_{K}+T_{M}$, (b) $T_{K}$, (c) $T_{M}$, and (d) $T_{M}^{H}$ for Hall parameters $\epsilon_{H}=0.05,0.025,0.0125$, and 0 (single-fluid MHD) are shown, respectively. All the transfer functions are normalized either by the use of $a_{\nu}$ and $\epsilon_{\nu}$ or by the use of $b_{\nu}$ and $\epsilon_{\eta}$. The transfer functions do not show clear dependence on the Hall parameter $\epsilon_{H}$ except the $T_{M}^{H}$, in which $\epsilon_{H}$ appears explicitly. However, we can see that $T_{M}^{H}$ collapse each other better if we normalize it by $\epsilon_{H}$. While $T_{K}(k)$ of $\epsilon_{H}=0.05$ is different from the other at $k a_{\eta} \leq 0.01$ and $k a_{\eta} \simeq 0.03$, it is not sufficiently large to make a clear difference in the energy spectra as we have seen in Fig. 2.

In Fig. 9, the transfer functions (a) $T_{K}$, (b) $T_{M}$, and (c) $T_{M}^{I}$ constructed by the filtered velocity field $\left(\bar{u}_{i}\right)$ and the filtered magnetic field $\left(\bar{B}_{i}\right)$, where ${ }^{-}$denotes the filtered (grid-scale,
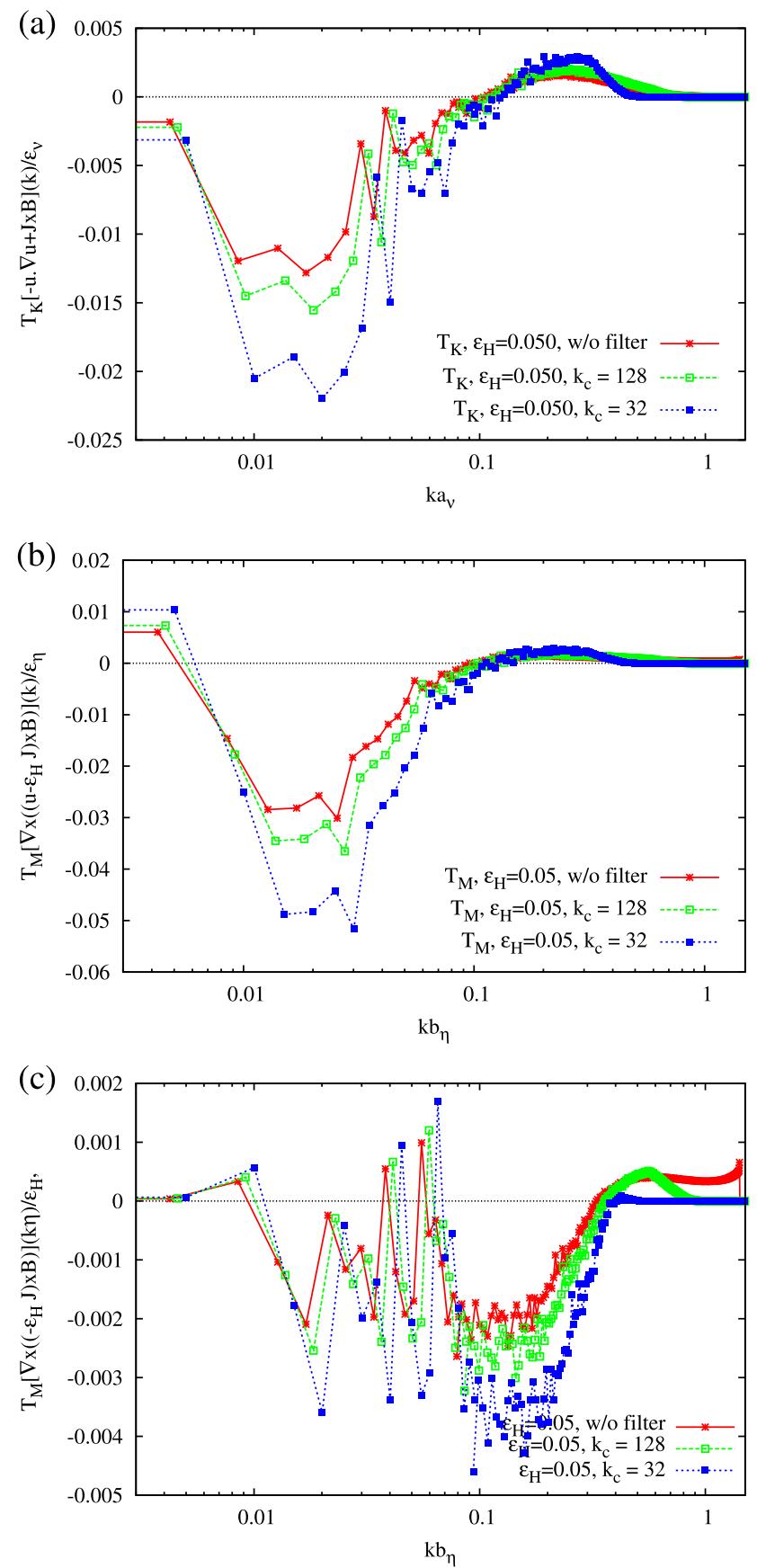

FIG. 9. Response of the transfer functions of $\epsilon_{H}=0.05$ Hall MHD turbulence to the low-pass filters.
GS) component, are presented for $\epsilon_{H}=0.05$. All the three transfer functions in Fig. 9 show that the amplitude of them is increased for smaller $k_{c}$. This suggests that the nonlinear coupling of the Fourier coefficients of $k>k_{c}$ and $k \leq k_{c}$ causes strong forward transfer to the wave number region $k>k_{c}$. However, there is a difference between $T_{K}, T_{M}$ and $T_{H}$. In Figs. 9(a) and 9(b), the amplitudes of the transfer functions are more increased for a smaller $k_{c}$ over a wide region of $k<k_{c}$. On the other hand in Fig. 9(c), the transfer function is amplified for a relatively narrow region of $0.1<k b_{\eta}<0.3$, and amplitudes at $k b_{\eta}<0.1$ are not influenced very much. This suggests that the nonlinear coupling of the Hall term is more local in the wave number space than $T_{K}$ and $T_{M}$, or, $T_{M}^{I}$ which is the difference between $T_{M}$ and $T_{M}^{I}$ by definition. This also means that the direct influence of the Hall term to dynamics of the scale larger than the ion skin depth can be limited to the relatively narrow range of $k<k_{c}$. Then, the indirect influence of the Hall term to the vortex structure can be limited to the scale slightly larger than the ion skin depth. This is consistent with the observation in Fig. 7 in which both the vortex sheet and the tubes are observed for $k_{c}=32$ visualization. An understanding consistent with the observation is that some regions are influenced locally by the Hall term and the structure transition is caused, while some regions remain without transition because very large scales are less influenced in comparison to small scales.

\section{PROJECTION OF THE SMALL SCALES TO A MODELED FIELD}

We have seen in Sec. IV that the forward energy transfer can be dominant in the nonlinear couplings. Then, the small scale dynamics might be simply substituted by a model which works to absorb the energy transfer from the large scales, as we often expect in a sub-grid-scale model for a Large Eddy Simulation (LES). In this section, we study how much nonlinear terms in the Hall MHD equations can be modeled by the use of a Smagorinsky-type diffusive model. The filtered (GS) equations are

$$
\begin{aligned}
\frac{\partial \bar{u}_{i}}{\partial t}= & -\frac{\partial}{\partial x_{j}}\left(\bar{u}_{i} \bar{u}_{j}-\bar{B}_{i} \bar{B}_{j}\right)-\frac{\partial}{\partial x_{i}}\left(\bar{p}+\frac{1}{2} \bar{B}_{j} \bar{B}_{j}\right) \\
& +\frac{1}{R e_{\nu}} \frac{\partial^{2} \bar{u}_{i}}{\partial x_{j} \partial x_{j}}+F_{i}^{1}
\end{aligned}
$$

$$
\begin{aligned}
\frac{\partial \bar{B}_{i}}{\partial t}=\epsilon_{i j k} \frac{\partial}{\partial x_{j}}\left[\epsilon_{k m n}\left(\bar{u}_{m}-\epsilon_{H} \bar{J}_{m}\right) \bar{B}_{n}-\frac{1}{R e_{\eta}} \bar{J}_{k}\right] \\
+F_{i}^{2}+F_{i}^{3}, \\
\frac{\partial \bar{u}_{i}}{\partial x_{i}}=0 \\
\frac{\partial \bar{B}_{i}}{\partial x_{i}}=0 .
\end{aligned}
$$

Here,

$$
F_{i}^{1}=-\frac{\partial}{\partial x_{j}}\left[\left(\overline{u_{i} u_{j}}-\overline{B_{i} B_{j}}\right)-\left(\bar{u}_{i} \bar{u}_{j}-\bar{B}_{i} \bar{B}_{j}\right)\right],
$$




$$
\begin{gathered}
F_{i}^{2}=\epsilon_{i j k} \frac{\partial}{\partial x_{j}}\left[\epsilon_{k m n}\left(\overline{u_{m} B_{n}}-\bar{u}_{m} \bar{B}_{n}\right)\right], \\
F_{i}^{3}=\epsilon_{i j k} \frac{\partial}{\partial x_{j}}\left[-\epsilon_{H} \epsilon_{k m n}\left(\overline{J_{m} B_{n}}-\bar{J}_{m} \bar{B}_{n}\right)\right]
\end{gathered}
$$

in Eqs. (9) and (10) are effects with SGS components. Instead of carrying out LES, we study how much these terms can be replaced by a Smagorinsky-type diffusivity.

In this article, we adopt a model proposed by Hamba and Tsuchiya ${ }^{40}$ (hereafter refereed to as the HT model), that is

$$
\begin{gathered}
\hat{F}_{i}^{1}=\frac{\partial}{\partial x_{j}}\left(\mu_{S G S} \bar{S}_{i j}\right), \\
\hat{F}_{i}^{2}=\epsilon_{i j k} \frac{\partial}{\partial x_{j}}\left(\mu_{S G S} \bar{J}_{i}\right), \\
\mu_{S G S}=\Delta^{2}\left(\frac{1}{2} \bar{S}_{i j} \bar{S}_{i j}+\frac{C_{\eta}}{C_{\nu}} \bar{J}_{i} \bar{J}_{i}\right)^{1 / 2}, \\
\bar{S}_{i j}=\frac{\partial \bar{u}_{i}}{\partial x_{j}}+\frac{\partial \bar{u}_{j}}{\partial x_{i}},
\end{gathered}
$$

where the symbol $\Delta$ denotes the filter width. Additionally, we attempt to apply the HT model to the Hall term as

$$
\hat{F}_{i}^{3}=-\epsilon_{H} \epsilon_{i j k} \frac{\partial}{\partial x_{j}}\left[\mu_{S G S}\left(\alpha \bar{S}_{i j}+\beta \bar{J}_{j}\right)\right] .
$$

The symbols $C_{\nu}$ and $C_{\eta}$ are the Smagorinsky constants for the viscosity and the resistivity. (We can show the results of LES of with the HT model, which reproduces the turbulent energy spectra fairly well.) We do neither need a precise value of $C_{\nu}, \alpha$ nor $\beta$ because we do not carry out LES here but rather project the DNS data to the model and adjust these coefficients so that the model can work most effectively to the turbulent field. Modeling of the Hall term with a theoretical base, including optimization of the two coefficients, is left to the next work.

Next, we introduce the norms

$$
\begin{gathered}
\Delta F^{i}=\left\langle\left|\mathbf{F}^{i}-\left(\mathbf{F}^{i} \cdot \hat{\mathbf{e}}^{i}\right) \hat{\mathbf{e}}^{i}\right|^{2}\right\rangle /\left\langle\left|\mathbf{F}^{i}\right|^{2}\right\rangle, \\
\hat{\mathbf{e}}^{i}=\hat{\mathbf{F}}^{i} /\left|\hat{\mathbf{F}}^{i}\right|
\end{gathered}
$$

as a measure to evaluate non-diffusive parts which cannot be modeled by the HT model in the SGS terms $\mathbf{F}^{i}(i=1,2,3)$ in DNS data. The symbol ${ }^{\wedge}$ is put on the SGS terms by the use of the HT model so that they can be distinguished from those in the DNS data. The essence of this measure, which is introduced in our previous work $^{33}$ first, is that $\left|\mathbf{F}^{i}-\left(\mathbf{F}^{i} \cdot \hat{\mathbf{e}}^{i}\right) \hat{\mathbf{e}}^{i}\right|^{2} \simeq 0$ if the SGS model mimics the SGS contributions to the GS components very well, while the behavior is not trivial for various $k_{c}$ because the measure is normalized by $\left\langle\left|\mathbf{F}^{i}\right|^{2}\right\rangle$. In Fig. 10, (a) $\Delta F^{1}$, (b) $\Delta F^{2}$, and (c)
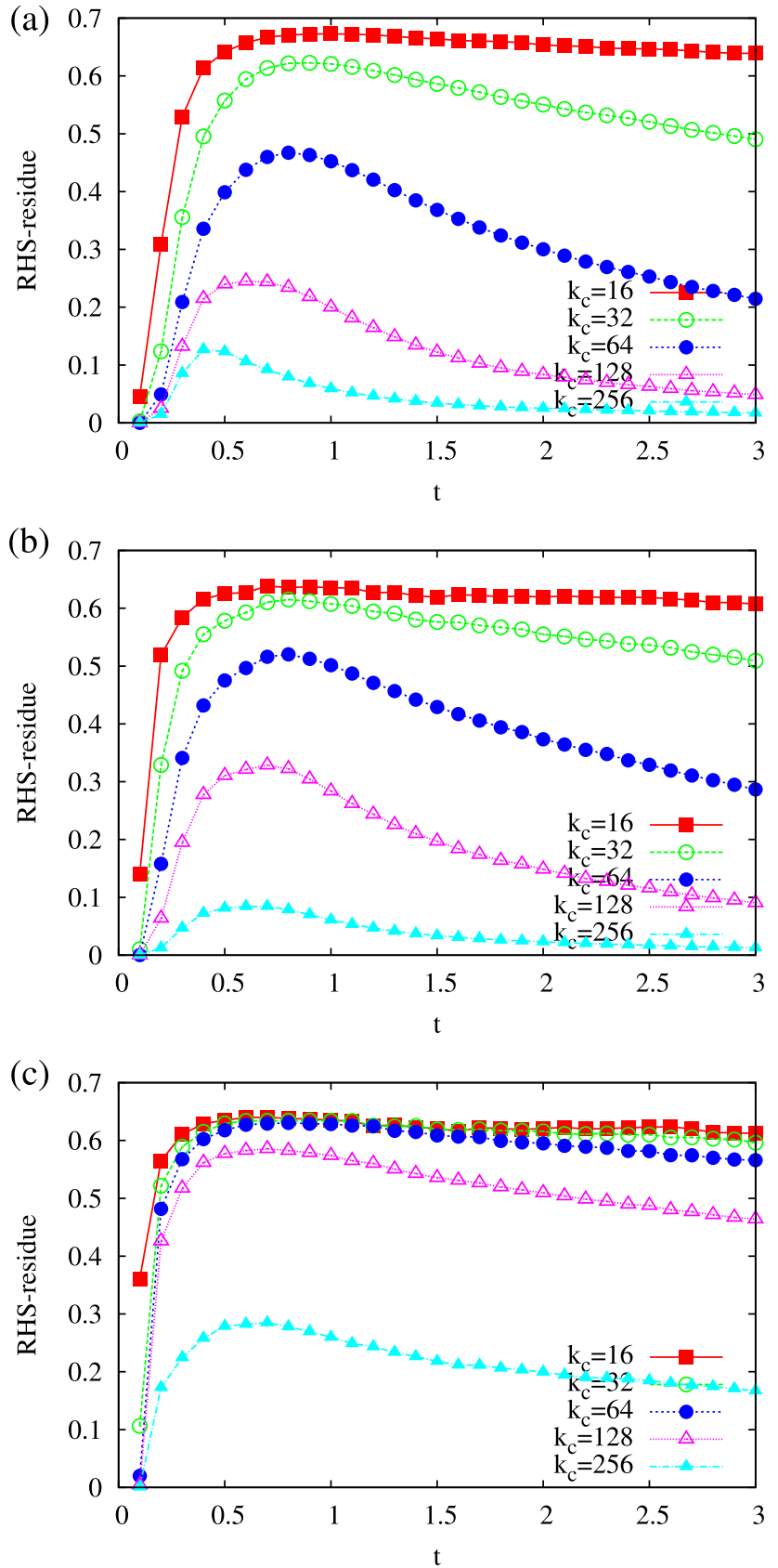

FIG. 10. The time evolution of the RHS-residues (a) $\Delta F^{1}$, (b) $\Delta F^{2}$, and (c) $\Delta F^{3}$ in Hall MHD turbulence with $\epsilon_{H}=0.05$

$\Delta F^{2}$ in Hall MHD and single-fluid MHD simulations are shown for the low-pass filter of various $k_{c}$ s, while their counterpart in the single-fluid MHD turbulence is shown in Fig. 11. All the plots in Figs.10 and 11 but Fig. 10(c) show that the residues are decreased quickly for a larger $k_{c}$. This suggests that the high wave number component of the SGS terms behaves diffusion-like. The plots of $\Delta F^{1}$ also show that there is no significant difference in the applicability of the HT model between Hall MHD and single-fluid MHD turbulence. However, Fig. 10(c) shows a salient difference from the others. The residue does not decrease for a larger $k_{c}$ for $k_{c}=128$, and it becomes small suddenly for $k_{c}=256$. It suggests that the SGS components of the Hall term can be approximated by a Smagorinsky-type diffusion model 

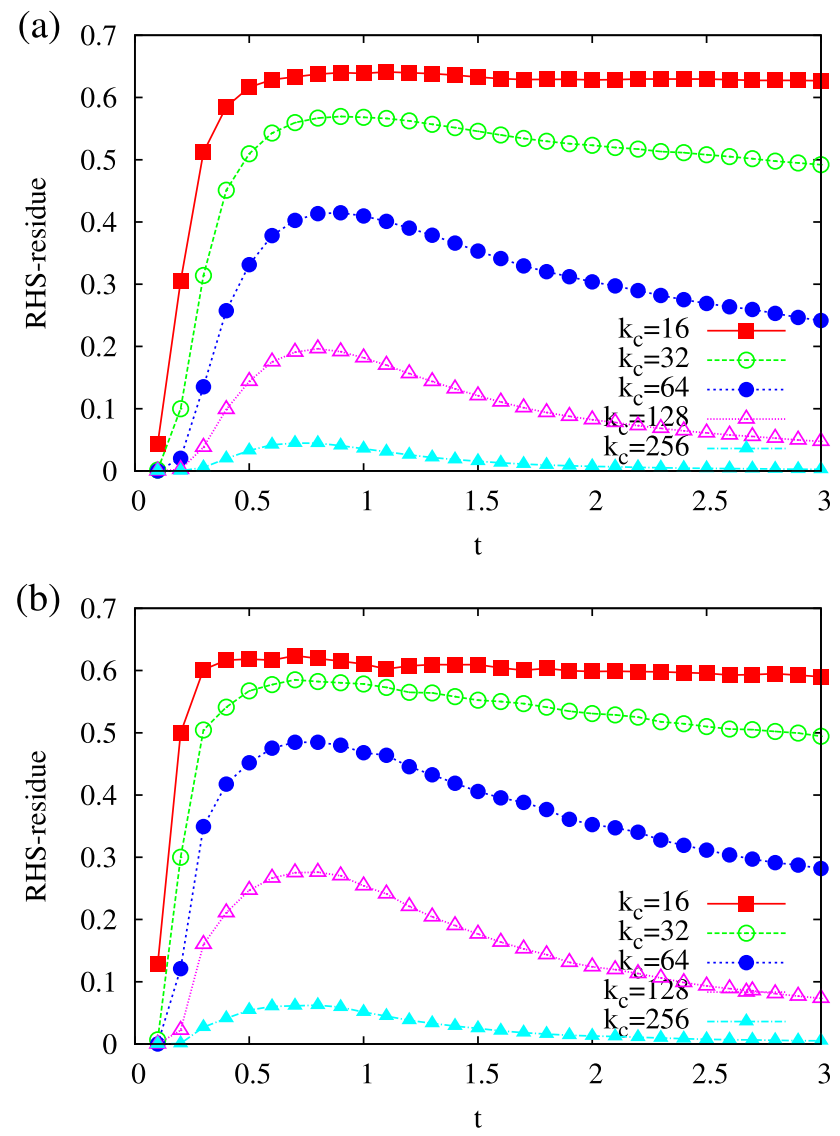

FIG. 11. The time evolution of the RHS-residues (a) $\Delta F^{1}$ and (b) $\Delta F^{2}$ in single-fluid MHD turbulence.

effectively for high wave numbers, and the scales $k<k_{c}=$ 128 can be dominated by non-diffusive motions. Eliminating the high wave number components can be important when we carry out LES of Hall MHD turbulence because the high wave number components in the Hall term can bring about the whistler waves with very high frequency and limit the time step width severely. It is also remarked that the separation between the plot of $k_{c}=256$ and the others in Fig. 10(c) suggests that there can be an intrinsic length scale in between $k=128$ and $k=256$ which separates non-diffusive and diffusive motions, although we have not clarified what the scale is. Further analysis on the scale-separation and its possible impacts to SGS-modeling will be shown elsewhere with results of LES. We may also need to take influences of compressibility, which has been modeled by Chernyshov et al., ${ }^{41}$ for example, into account there.

\section{CONCLUDING REMARKS}

We have studied influences of the Hall term to the local structure in MHD turbulence. It is found that transition of vortices from sheet-like to tubular structures is induced by the Hall term, and that the energy spectrum does not distinguish the sheet and tubular structures. The sharp low-pass filters with various cut-off wave numbers are utilized to see influences of the Hall term among some scales. It is shown that the transition of the vortex structures can occur not only in the scales smaller than the ion skin depth but also in a larger scale. The nonlinear energy transfer by the Hall term appears forward-transfer-dominant and local in the wave number space. An analysis by the use of a Smagorinsky-type SGS model suggests that only a high wave number component of the Hall term can be replaced by the model effectively. This also suggests that there can be an intrinsic scale which separates the moderate and high wave numbers components.

We have concentrated on the structure transition and have not shown turbulence statistics in this article. However, of course, turbulence statistics and structures are closely related with each other. For example, the alignment of the velocity and the magnetic field vectors, as has been studied by Matthaeus et al., ${ }^{26}$ are under the strong influence of the Hall effect and should be related to the structure transition. Also, we have only seen energy transfer functions quickly and have skipped discussions about locality and non-locality in the wave number space and in the real space. Detailed reports on the statistics and the energy transfer functions will be shown in succeeding papers.

\section{ACKNOWLEDGMENTS}

One of the authors (H.M.) would like to express sincere thanks to Professor F. Hamba and Professor N. Yokoi of The University of Tokyo and Professor T. Gotoh of Nagoya Institute of Technology for their suggestions. The numerical simulations were performed on HITACHI SR16000 Plasma Simulator of National Institute for Fusion Science (NIFS), NEC SX-9 of Tohoku University, and Fujitsu FX-10 of Nagoya University. This research was partially supported by the NIFS Collaborative Research Program (Nos. NIFS10KTAS001, NIFS09KNXN154, NIFS10KTBS003, and NIFS11KNS0016), Grant-in-Aid for Scientific Research, KAKENHI (Nos. 22540509, 23340182, and 23540583), and the HPC project of Nagoya University.

${ }^{1}$ P. S. Iroshnikov, Sov. Astron. 7, 566-571 (1964).

${ }^{2}$ R. H. Kraichnan, Phys. Fluids 8, 1385 (1965).

${ }^{3}$ S. Sridhar and P. Goldreich, Astrophys. J. 432, 612 (1994).

${ }^{4}$ P. Goldreich and S. Sridhar, Astrophys. J. 485, 680 (1997).

${ }^{5}$ O. Stawicki, S. Peter Gary, and H. Li, J. Geophys. Res. 106, A8273-A8281, doi:10.1029/2000JA000446 (2001).

${ }^{6}$ J. Cho, A. Lazarian, and E. T. Vishniac, Astrophys. J. 566, L49 (2002).

${ }^{7}$ S. D. Bale, P. J. Kellogg, F. S. Mozer, T. S. Horbury, and H. Reme, Phys. Rev. Lett. 94, 215002 (2005).

${ }^{8}$ T. S. Horbury, M. Forman, and S. Oughton, Phys. Rev. Lett. 101, 175005 (2008).

${ }^{9}$ O. Alexandrova, J. Saur, C. Lacombe, A. Mangeney, J. Mitchell, S. J. Schwartz, and P. Robert, Phys. Rev. Lett. 103, 165003 (2009).

${ }^{10}$ K. H. Kiyani, S. C. Chapman, Yu. V. Khotyaintsev, M. W. Dunlop, and F. Sahraoui, Phys. Rev. Lett. 103, 075006 (2009).

${ }^{11}$ S. M. Mahajan and Z. Yoshida, Phys. Plasmas 7, 635 (2000)

${ }^{12}$ L. E. Sugiyama and W. Park, Phys. Plasmas 7, 4644 (2000).

${ }^{13}$ J. D. Huba and D. Winske, Phys. Plasmas 5, 2305 (1998).

${ }^{14}$ P. Zhu, D. D. Schnack, F. Erahimi, E. G. Zweibel, M. Suzuki, C. C. Hegna, and C. R. Sovinec, Phys. Rev. Lett. 101, 085005 (2008).

${ }^{15}$ R. Goto, H. Miura, A. Ito, S. Masahiko, and T. Hatori, Plasma Fusion Res. 9, 1403076 (2014).

${ }^{16}$ K. Yamamoto and I. Hosokawa, J. Phys. Soc. Jpn. 57, 1532-1535 (1988).

${ }^{17}$ H. Miura, J. Turbulence 5, N10 (2004).

${ }^{18}$ Y. Kaneda and T. Ishihara, J. Turbulence 7, N20 (2006).

${ }^{19}$ D. Biskamp, Magnetohydrodynamic Turbulence (Cambridge University Press, 2003).

${ }^{20}$ A. Alexakis, P. D. Minini, and A. Pouquet, New J. Phys. 9, 298 (2007). 
${ }^{21}$ K. Yoshimatsu, Y. Kondo, K. Schneider, N. Okamoto, H. Hagiwara, and M. Farge, Phys. Plasmas 16, 082306 (2009).

${ }^{22}$ P. D. Mininni, D. O. Gom'ez, and S. M. Mahajan, Astrophys. J. 587, 472 (2003).

${ }^{23}$ P. D. Mininni, D. O. Gom'ez, and S. M. Mahajan, Astrophys. J. 619, 1019 (2005).

${ }^{24}$ P. Mininni, A. Alexakis, and A. Pouquet, J. Plasma Phys. 73, 377-401 (2007).

${ }^{25}$ P. Dmitruk and W. H. Matthaeus, Phys. Plasmas 13, 042307 (2006).

${ }^{26}$ W. H. Matthaeus, A. Pouquet, P. D. Mininni, P. Dmitruk, and B. Breech, Phys. Rev. Lett. 100, 085003 (2008).

${ }^{27}$ S. Servidio, W. H. Matthaeus, and P. Dmitruk, Phys. Rev. Lett. 100, 095005 (2008).

${ }^{28}$ P. Rodriguez Imazio, L. N. Martin, P. Dmitruk, and P. D. Mininni, Phys. Plasmas 20, 052506 (2013).
${ }^{29}$ K. Araki and H. Miura, Plasma Fusion Res. 8, 2401137 (2013).

${ }^{30}$ D. Hori and H. Miura, Plasma Fusion Res. 3, S1053 (2008).

${ }^{31}$ H. Miura, J. Plasma Fusion. Res. 5, S2059 (2010).

${ }^{32} \mathrm{~K}$. Araki and H. Miura, Plasma Fusion Res. 5, S2048 (2010).

${ }^{33} \mathrm{H}$. Miura and K. Araki, Plasma Phys. Controlled Fusion 55, 014012 (2013).

${ }^{34}$ W. H. Matthaeus, P. Dmitruk, D. Smith, S. Ghosh, and S. Oughton, Geophys. Res. Lett. 30, 2104, doi:10.1029/2003GL017949 (2003).

${ }^{35}$ S. Galtier and E. Buchlin, Astrophys. J. 656, 560-566 (2007).

${ }^{36}$ S. Kida and H. Miura, J. Phys. Soc. Jpn. 69, 3466 (2000).

${ }^{37}$ H. Miura and S. Kida, J. Phys. Soc. Jpn. 66, 1331 (1997)

${ }^{38}$ S. Kida and H. Miura, J. Phys. Soc. Jpn. 67, 2166 (1998).

${ }^{39}$ S. Kida and H. Miura, Euro. J. Mech. B/Fluids 17, 471 (1998).

${ }^{40}$ F. Hamba and M. Tsuchiya, Phys. Plasmas 17, 012301 (2010).

${ }^{41}$ A. A. Chernyshov, K. V. Kalesky, and A. Petrosyan, Phys. Plasmas 13, 032304 (2006). 